\title{
Continuum discretization in a basis of transformed harmonic-oscillator states
}

\author{
F. Pérez-Bernal, ${ }^{1}$ I. Martel,${ }^{1}$ J. M. Arias, ${ }^{2}$ and J. Gómez-Camacho ${ }^{2}$ \\ ${ }^{1}$ Departamento de Física Aplicada e Ingeniería Eléctrica, Universidad de Huelva, 21071 Huelva, Spain \\ ${ }^{2}$ Departamento de Física Atómica, Molecular y Nuclear, Facultad de Física, Universidad de Sevilla, Apartado 1065, \\ 41080 Sevilla, Spain
}

(Received 10 November 2000; published 18 April 2001)

\begin{abstract}
The inclusion of the continuum in the study of weakly bound systems is discussed. A transformed harmonicoscillator basis is introduced to provide an appropriate discrete and finite basis for treating the continuum part of the spectrum. As examples of application of the method the one-dimensional Poeschl-Teller and Morse potentials are worked out. The strength functions corresponding to different operators that couple the ground state to the continuum are investigated. It is found that the energy moments of those distributions are accurately reproduced with a small basis set.
\end{abstract}

DOI: 10.1103/PhysRevA.63.052111

PACS number(s): 03.65.Ca, 21.60.-n, 31.15.-p, 34.10.+x

\section{INTRODUCTION}

A general time-independent quantum-mechanical potential gives rise to a Hamiltonian with both bound and unbound eigenstates. Usually, the Hamiltonian of the system has a finite number of bound eigenstates while the unbound ones form a continuum. Therefore, a calculation of the system properties in terms of eigenfunctions of $H$ involves a summation over the discrete states as well as an integration over the continuum ones. The last one is an involved task and normally the properties of the bound system are analyzed by just using the bound eigenstates, while the continuum ones are of special relevance for dispersion processes. However, the study of the effect of the continuum part of the spectrum for treating properties of the bound system has a long tradition in physics (conversely, the effect of the bound states on dispersion processes has been widely investigated too). Recent examples can be found in nuclear [1-6], molecular [7-9], and atomic physics [10-13]. In particular, in nuclear physics the advent of the radioactive beam facilities has provided a variety of new nuclear structure problems [14] that include halo nuclei and neutron and proton rich nuclei close to the drip lines. All these systems are weakly bound and their proper treatment requires the inclusion in some way of the continuum part of the spectrum. This has been done in several ways, each one having its own advantages and drawbacks. Among them we cite:

(i) The $R$-matrix method [15] in which the basic idea is to solve the many-body problem in a box and then make the matching with the adequate boundary conditions.

(ii) The use of a Sturmian basis [16-18], where one uses bound states of scaled potentials, which are orthogonal when weighted with the potentials.

(iii) The Siegert pseudostate formulation [19], which provides a finite basis representation of the outgoing wave solutions to the radial Schrödinger equation for cutoff potentials.

(iv) The use of Gamow states [20], which are nonnormalizable solutions of the Schrödinger equation corresponding to outgoing boundary conditions characterized by complex energies.

(v) The method of continuum discretization coupled chan- nels (CDCC) [21] in which the continuum is discretized by means of taking fixed intervals, or bins, of $k$-values in the continuum states.

(vi) The expansion of the single-particle wave functions in a harmonic-oscillator basis [22].

This last method has become very popular since it provides a simple complete discrete basis. However, for weakly bound systems the Gaussian asymptotic behavior of the harmonic-oscillator wave functions is a poor representation of the continuum. Thus, methods based in a general localscaling point transformation to the harmonic-oscillator functions [23-27] have drawn considerable attention recently [1-3]. The so-called transformed harmonic-oscillator basis (THO) retains the simplicity of the harmonic-oscillator expansion and includes the correct asymptotic behavior.

In this paper we discuss a way of defining a THO basis designed to take into account the continuum by an appropriate discretization. We present the method and apply it to two analytic one-dimensional potentials of interest in molecular physics: the Morse potential and the Poeschl-Teller potential. The method can be equally applied to three-dimensional potentials. The paper is structured as follows. First, in Sec. II, the formalism is presented and the transformation to introduce a THO basis is proposed. In Sec. III, the application of the formalism to the Poeschl-Teller and Morse potentials is worked out. Finally, in Sec. IV the outlook and conclusions of this paper are presented.

\section{FORMALISM OF THE TRANSFORMED HARMONIC- OSCILLATOR STATES (THO) IN ONE-DIMENSIONAL HAMILTONIANS}

In this section we will apply the formalism of transformed harmonic-oscillator states to weakly bound systems. Such systems as the deuteron, halo nuclei, or Van der Waals molecules are of current interest. We consider the onedimensional Hamiltonian given by

$$
h=-\frac{\hbar^{2}}{2 \mu} \frac{d^{2}}{d r^{2}}+v(r),
$$

where $r$ is the relative coordinate of two particles, $\mu$ is the reduced mass, and $v(r)$ is the interaction between both par- 
ticles. If distances are measured in units of $\alpha^{-1}$ such that $x$ $=\alpha r$ is dimensionless and energies are given in units of $\hbar^{2} \alpha^{2} / \mu$, the preceding Hamiltonian can be written as

$$
h=-\frac{1}{2} \frac{d^{2}}{d x^{2}}+v(x)
$$

which will be the Hamiltonian used hereafter. Note that $h$ and $v(x)$ are then dimensionless quantities.

In order to maximize the continuum contribution we assume that the system has just one bound state, $\psi_{B}(x)$, though the present formalism can be easily extended to systems with several bound states as well as to three-dimensional systems:

$$
h \psi_{B}(x)=e_{B} \psi_{B}(x) \text {. }
$$

The Hamiltonian $h$ has also an infinite number of eigenstates in the continuum that, however, are not normalizable. Our objective is to develop a procedure that allows a convenient description of the states in the continuum by means of a finite number of normalizable states.

The general formalism presented herewith is applied in the next section to two cases of interest in molecular physics, the Poeschl-Teller [28], and Morse [29] potentials.

\section{A. Coordinate transformation}

Let us consider the one dimensional harmonic-oscillator basis

$$
\phi_{n}^{H O}(s)=\mathcal{N}_{n} H_{n}(s) \exp \left(-s^{2} / 2\right),
$$

where $H_{n}(s)$ are the usual Hermite polynomials and $\mathcal{N}_{n}$ $=\left(\sqrt{\pi} 2^{n} n !\right)^{-1 / 2}$ the corresponding normalization constants. This basis is orthogonal and forms a complete set for all functions that are square integrable in $s$. Besides, if we make an arbitrary change of coordinates, given by the monotonously increasing function $x=x(s)$, and its inverse $s=s(x)$, the functions

$$
\varphi_{n}^{T H O}(x)=\sqrt{\frac{d s}{d x}} \phi_{n}^{H O}[s(x)]
$$

are orthogonal and form a complete set of all the functions that are square integrable in $x$. These functions are called THO states.

The transformation $x(s)$ is arbitrary in principle. However, it can be chosen in order to describe properly the properties of bound states in finite potentials. So, for small values of $s$, the harmonic-oscillator may be a reasonable approximation for the potential $v(x)$, and thus $x$ should depend linearly on $s$. However, for large values of $s$, the harmonic-oscillator wave functions behave as $\exp \left(-s^{2} / 2\right)$, while the bound wave function in $v(x)$ behaves as $\exp (-q x)$, where $q^{2} / 2=e_{B}$. So, for large $s, q x$ has to be proportional to $s^{2} / 2$.

If the bound state wave function $\psi_{B}(x)$ is known, the transformation $x(s)$ can be completely determined by requiring that

$$
\varphi_{0}^{T H O}(x)=\psi_{B}(x)
$$

This condition together with Eq. (5) provide a basis set with the asymptotic behavior described above. Equation (6) is equivalent to the nonlinear equation

$$
\int_{-\infty}^{x}\left|\psi_{B}\left(x^{\prime}\right)\right|^{2} d x^{\prime}=\int_{-\infty}^{s}\left|\phi_{0}^{H O}\left(s^{\prime}\right)\right|^{2} d s^{\prime}=\frac{1+\operatorname{erf}(s)}{2},
$$

which defines implicitly the function $x=x(s)$ as well as its inverse. It should be noticed that the derivative can be written as

$$
\frac{d x(s)}{d s}=\left(\frac{\phi_{0}^{H O}(s)}{\psi_{B}[x(s)]}\right)^{2}
$$

Once the wave function of the ground state provides the function $x=x(s)$, we can employ the THO basis, Eq. (5), to describe the continuum of our system. Note that, as the THO are orthogonal, and the $n=0$ state is the only bound state of the system, the states with $n \geqslant 1$ describe the continuum. We expect that, as the dimension of the THO basis increases, the wave functions explore distances beyond the range of the potential and, at the same time, they have oscillations inside the potential. Thus, the THO basis allows for an appropriate description of long range phenomena, and at the same time it permits to describe accurately short-range effects.

\section{B. Diagonalization of the Hamiltonian. Width of the states.}

We evaluate the matrix elements of the Hamiltonian $h$ in the THO basis. It should be noticed that the state $\varphi_{0}^{T H O}(x)$ $=\psi_{B}(x)$ is an eigenstate of $h$, but this is not the case for the states with $n \geqslant 1$. Let us consider the matrix element

$$
\begin{aligned}
& \left\langle\mathrm{THO}, n\left|\left(h-e_{B}\right)\right| \mathrm{THO}, m\right\rangle \\
& \quad=\int d x \varphi_{n}^{T H O}(x)\left(h-e_{B}\right) \varphi_{m}^{T H O}(x) .
\end{aligned}
$$

We can take into account that $\varphi_{m}^{\text {THO }}(x)$ $=\pi^{1 / 4} \mathcal{N}_{m} H_{m}[s(x)] \varphi_{0}^{T H O}(x)$, and that $\left(h-e_{B}\right) \varphi_{0}^{T H O}(x)=0$, to write

$$
\begin{aligned}
& \left\langle\mathrm{THO}, n\left|\left(h-e_{B}\right)\right| \mathrm{THO}, m\right\rangle \\
& =\frac{\sqrt{\pi} \mathcal{N}_{n} \mathcal{N}_{m}}{2} \int d x \varphi_{0}^{T H O}(x)\left[H_{n}[s(x)],\right. \\
& \left.\left[\left(h-e_{B}\right), H_{m}[s(x)]\right]\right] \varphi_{0}^{T H O}(x) .
\end{aligned}
$$

The double commutator is independent of the potential, and gives

$$
\left[H_{n}[s(x)],\left[\left(h-e_{B}\right), H_{m}[s(x)]\right]\right]=\frac{d H_{n}[s(x)]}{d x} \frac{d H_{m}[s(x)]}{d x} ;
$$

writing the integral in terms of $s$, one gets 


$$
\begin{aligned}
& \left\langle\mathrm{THO}, n\left|\left(h-e_{B}\right)\right| \mathrm{THO}, m\right\rangle \\
& \quad=2 n \mathcal{N}_{n} m \mathcal{N}_{m} \int d s \exp \left(-s^{2}\right) H_{n-1}(s) H_{m-1}(s)\left(\frac{d s}{d x}\right)^{2} .
\end{aligned}
$$

This expression can be easily evaluated using Gaussian quadratures. Note that the only information required is the derivative of the function $x(s)$, evaluated at the points $s_{n}$, which define the quadrature.

The matrix elements with $n=0$ or $m=0$ vanish. This is due to the fact that the state of $n=0$ is an eigenstate of the Hamiltonian. Let us consider that we diagonalize the Hamiltonian in a $N$ dimensional basis of THO states, from $i=0$ to $i=N-1$. The eigenstates of the Hamiltonian, in this restricted basis, are given by

$$
\begin{gathered}
|N, 0\rangle=|\mathrm{THO}, 0\rangle \\
|N, i\rangle=\sum_{j=1}^{N-1}|\mathrm{THO}, j\rangle\langle\mathrm{THO}, j \mid N, i\rangle,
\end{gathered}
$$

where the states $|N, i\rangle(i=1, \ldots, N-1)$ represent the continuum states in the truncated $N$ dimensional THO basis. They can be expressed in the $x$ representation as

$$
\langle x \mid N, i\rangle=\psi_{i}^{N}(x)=\pi^{1 / 4} P_{i}^{N-1}[s(x)] \varphi_{0}^{T H O}(x),
$$

where $P_{i}^{N-1}(s)$ is a polynomial given by

$$
P_{i}^{N-1}(s)=\sum_{j=1}^{N-1} \mathcal{N}_{j} H_{j}(s)\langle\mathrm{THO}, j \mid N, i\rangle .
$$

The eigenvalues of the Hamiltonian, in the restricted basis, are related to the wave function through

$$
\begin{aligned}
\left(e_{i}^{N}-e_{B}\right)= & \frac{1}{2} \delta_{i j} \int d s \exp \left(-s^{2}\right) \\
& \times \frac{d P_{i}^{N-1}(s)}{d s} \frac{d P_{j}^{N-1}(s)}{d s}\left(\frac{d s}{d x}\right)^{2} .
\end{aligned}
$$

The use of the THO basis also allows to calculate the width of the states. In order to do so, we evaluate the matrix elements of the operator $\left(h-e_{B}\right)^{2}$ in the basis $|N, i\rangle$. Note that if the states $|N, i\rangle$ were the true eigenstates of the Hamiltonian, in a complete basis, then this matrix element would just be $\left(E_{i}^{N}-e_{B}\right)^{2}$. However, as $|N, i\rangle$ are only eigenstates of the Hamiltonian in a restricted basis, they will show a spread of energies when expanded in terms of the true continuum eigenstates of $h$. A measure of that spread is given by

$$
\Gamma_{i}^{N}=\sqrt{\left\langle N, i\left|\left(h-e_{B}\right)^{2}\right| N, i\right\rangle-\left(E_{i}^{N}-e_{B}\right)^{2}} .
$$

Let us use the fact that the THO states form a complete basis. Then, we have

$$
\begin{aligned}
& \left\langle N, i\left|\left(h-e_{B}\right)^{2}\right| N, i\right\rangle \\
& =\sum_{n=0}^{\infty}\left\langle N, i\left|h-e_{B}\right| \mathrm{THO}, n\right\rangle\left\langle\mathrm{THO}, n\left|h-e_{B}\right| N, i\right\rangle .
\end{aligned}
$$

Using Eqs. (12) and (16)

$$
\begin{aligned}
& \left\langle\text { THO }, n\left|\left(h-e_{B}\right)\right| N, i\right\rangle \\
& \quad=\frac{1}{2} \mathcal{N}_{n} \int d s \exp \left(-s^{2}\right) \frac{d H_{n}(s)}{d s} \frac{d P_{i}^{N-1}(s)}{d s}\left(\frac{d s}{d x}\right)^{2} .
\end{aligned}
$$

This expression can be integrated by parts to give

$$
\begin{aligned}
\langle\text { THO, } & ,\left(h-e_{B}\right)|N, i\rangle \\
= & \frac{1}{2} \mathcal{N}_{n} \int d s H_{n}(s) \exp \left(-s^{2}\right)\left(2 s-\frac{d}{d s}\right) \\
& \times\left(\frac{d P_{i}^{N-1}(s)}{d s}\left(\frac{d s}{d x}\right)^{2}\right) .
\end{aligned}
$$

Now, we can use the closure properties of the Hermite polynomials, to obtain

$$
\begin{aligned}
\langle N, i| & \left(h-e_{B}\right)^{2}|N, i\rangle \\
= & \left(\frac{1}{2}\right)^{2} \int d s \exp \left(-s^{2}\right)\left[\left(2 s-\frac{d}{d s}\right)\right. \\
& \left.\times\left(\frac{d P_{i}^{N-1}(s)}{d s}\left(\frac{d s}{d x}\right)^{2}\right)\right]^{2} .
\end{aligned}
$$

It is remarkable that the knowledge of the function $x(s)$ is all we need to obtain wave functions, energies, and widths of the Hamiltonian eigenstates in the THO basis.

\section{Matrix elements of operators. Sum rules}

Let us consider the matrix elements of an arbitrary local operator $O(x)$, which is a function of the coordinate $x$. The matrix element that connects the ground state $|N, 0\rangle$ to the continuum state $|N, i\rangle$ is just

$$
\langle N, i|O| N, 0\rangle=\pi^{1 / 4} \int d x P_{i}^{N-1}[s(x)] O(x)\left|\varphi_{0}^{T H O}(x)\right|^{2} .
$$

This integral is more conveniently written in terms of the variable $s$

$$
\langle N, i|O| N, 0\rangle=\sqrt{\pi} \int d s P_{i}^{N-1}(s) O(x(s)) \exp \left(-s^{2}\right),
$$

an expression that can be very simply evaluated using Gaussian quadratures. From this last formula we can define the following global magnitudes.

(i) Total strength: It is given by 


$$
\mathcal{S}_{T}(O ; N)=\sum_{i}|\langle N, i|O| N, 0\rangle|^{2} .
$$

In the limit of very large number of states $N$, the set of states becomes a complete set, and one can use closure, so that

$$
\mathcal{S}_{T}(O)=\lim _{N \rightarrow \infty} \mathcal{S}_{T}(O ; N)=\left\langle N, 0\left|O^{2}\right| N, 0\right\rangle .
$$

So, one should obtain, in the large $N$ limit,

$$
\begin{aligned}
\mathcal{S}_{T}(O) & =\pi^{-1 / 2} \int d s O(x(s))^{2} \exp \left(-s^{2}\right) \\
& =\int d x O(x)^{2} \psi_{B}(x)^{2} .
\end{aligned}
$$

(ii) Energy weighted sum rule: It is given by

$$
\mathcal{E}_{W}(O ; N)=\sum_{i}\left(e_{i}^{N}-e_{B}\right)|\langle N, i|O| N, 0\rangle|^{2} .
$$

In the limit of very large number of states $N$, we can use the basis closure to express $\mathcal{E}_{W}(O)=\lim _{N \rightarrow \infty} \mathcal{E}_{W}(O ; N)$ in terms of a double commutator

$$
\mathcal{E}_{W}(O)=\frac{1}{2}\left\langle N, 0\left|\left[O(x),\left[h-e_{B}, O(x)\right]\right]\right| N, 0\right\rangle .
$$

The double commutator can be explicitly evaluated, to give

$$
\left[O(x),\left[h-e_{B}, O(x)\right]\right]=\left(\frac{d O(x)}{d x}\right)^{2} .
$$

Thus, one obtains

$$
\begin{aligned}
\mathcal{E}_{W}(O) & =\pi^{-1 / 2} \frac{1}{2} \int d s\left(\left.\frac{d O(x)}{d x}\right|_{x=x(s)}\right)^{2} \exp \left(-s^{2}\right) \\
& =\frac{1}{2} \int d x[d O(x) / d x]^{2} \psi_{B}(x)^{2}
\end{aligned}
$$

(iii) Polarizability: It is given by

$$
\mathcal{P}(O ; N)=\sum_{i \neq 0}\left(e_{i}^{N}-e_{B}\right)^{-1}|\langle N, i|O| N, 0\rangle|^{2} .
$$

In the limit of a large number of states, $\mathcal{P}(O)$ $=\lim _{N \rightarrow \infty} \mathcal{P}(O ; N)$ converges to a constant value, that can be evaluated from the variation of matrix element of the Hamiltonian $h-e_{B}$ with the ground state $|g s(t)\rangle$ of a perturbed Hamiltonian $h+t O(x)$ [30]:

$$
\mathcal{P}(O)=\frac{1}{2} \lim _{t \rightarrow 0} \frac{d^{2}}{d t^{2}}\left\langle g s(t)\left|h-e_{B}\right| g s(t)\right\rangle .
$$

We can use the values of these global magnitudes to evaluate the convergence as the number of THO states included in the calculation is increased. With regard to the form of the operators $O(x)$, we will consider two different cases. First, we take $O(x)=x$, a long-range operator, to describe effects of external fields, such as the Coulomb field. In this case, $x$ represents the electric dipole operator. In second place, we consider a short-range operator $O(x)=v(x)$, to describe possible effects of internal correlations, which would have a range similar to the potential.

\section{APPLICATION TO ANALYTIC ONE-DIMENSIONAL HAMILTONIANS}

\section{A. The Poeschl-Teller Hamiltonian}

The Poeschl-Teller potential [28] is widely used in molecular physics, for example, to model bending vibrations, and conveys a considerable attention in other fields [6]. It is written as

$$
v(x)=-D \frac{1}{\cosh ^{2}(x)},
$$

where $-D$ is the value of the potential in its minimum. The variable $x=\alpha r$, where $r$ is the relative coordinate and $\alpha$ is the inverse of the range of the potential. The depth of the potential $D$ can be written as

$$
D=\frac{1}{2} j(j+1),
$$

in terms of a new parameter $j$ [31] which is a positive real number. The bound eigenstates of the Poeschl-Teller Hamiltonian can be written in terms of $j$ as

$$
\Psi_{j v}(x)=\mathcal{N}_{j v} P_{j}^{(j-v)}(z),
$$

where $v$ is an integer taking values from 0 to the integer part of $j, \mathcal{N}_{j v}=\sqrt{(j-v) v ! /(2 j-v) !}$ is a normalization constant, $z=\tanh (x)$, and $P_{s}^{(p)}(z)$ are the associated Legendre functions when $s$ is integer. In the present paper we consider $j$ $=1$, the only true bound state $v=0$, has an energy of $e_{B}=$ $-\frac{1}{2}$ and its wave function is written as

$$
\psi_{B}^{P T}(x)=\frac{1}{\sqrt{2} \cosh (x)} .
$$

In this case there is another state for $v=1$, which is not normalizable, corresponding to a resonance in the continuum at zero energy. The use of an integer value of $j$ is assumed, in the present paper for simplicity, but it is not mandatory. The relationship between $x$ and $s$ stems from the Eq. (5)

$$
\frac{1}{\sqrt{2} \cosh (x)}=\sqrt{\frac{d s}{d x}} \pi^{-1 / 4} \exp \left(-s^{2} / 2\right) .
$$

Direct integration gives both the dependence of $s$ on $x$ and vice versa: $\operatorname{erf}(s)=\tanh (x)$. The $s(x)$ function is presented in Fig. 1.

In this case the derivative of the function $s(x)$ can be written in terms of the $s$ variable as 


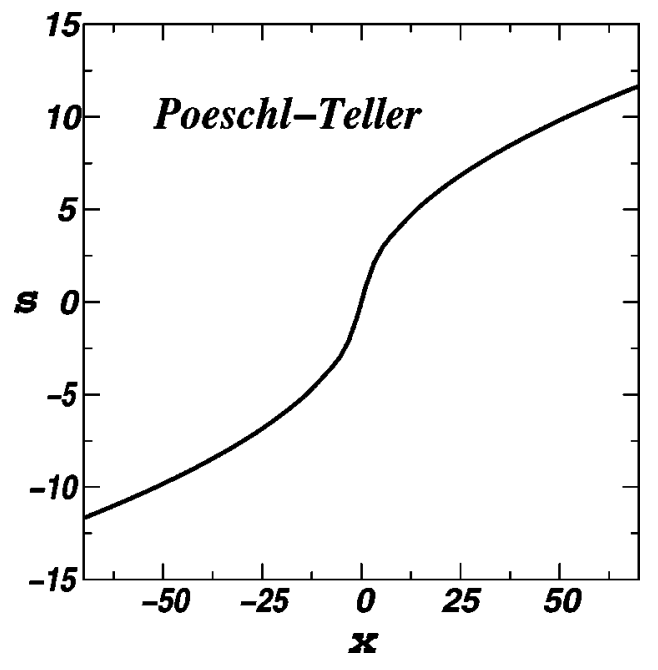

FIG. 1. Function $s(x)$ for the Poeschl-Teller Hamiltonian characterized by $j=1$.

$$
\frac{d s}{d x}=\frac{\sqrt{\pi}}{2} \exp \left(s^{2}\right)\left[1-\operatorname{erf}^{2}(s)\right]
$$

facilitating the calculations. This result allows us to write the THO basis in the $s$ coordinate space as

$$
\varphi_{n}^{T H O}(x)=\frac{1}{\sqrt{2^{n+1} n !}} H_{n}[s(x)] \sqrt{1-\operatorname{erf}^{2}[s(x)]} .
$$

In Fig. 2 we plot the first five states of the THO basis for $j$ $=1$. In this case the Hamiltonian matrix can be easily computed from Eq. (12) and its diagonalization provides us with eigenvalues and eigenfunctions. According to Eq. (6), we obtain one negative eigenvalue at the precise energy $e_{B}=$ $-\frac{1}{2}$, and in addition, $N-1$ positive eigenvalues corresponding to the continuum discretization. The resulting energies for increasing values of the $N$ parameter are depicted in Fig. 3 , with $N$ ranging from 2 to 20 . The appearance of symmetry doublets is due to the symmetric form of the potential, which provides wave functions with well-defined parity. As the di-

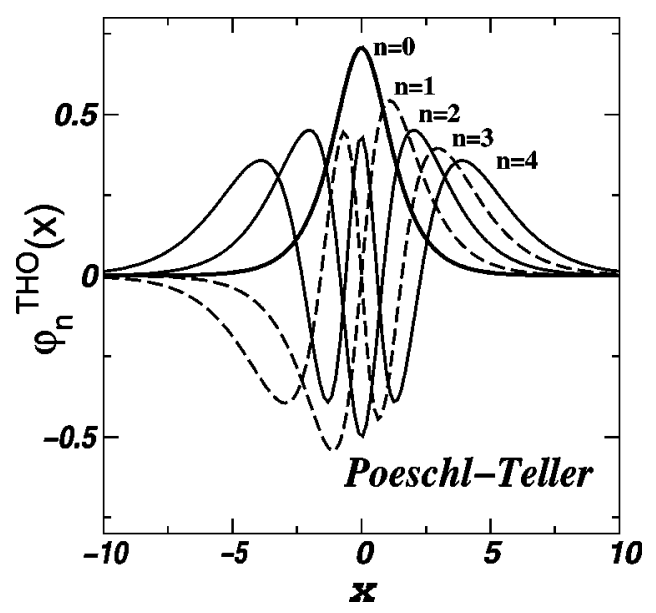

FIG. 2. THO basis for the Poeschl-Teller Hamiltonian, from $n$ $=0$ to 4 .

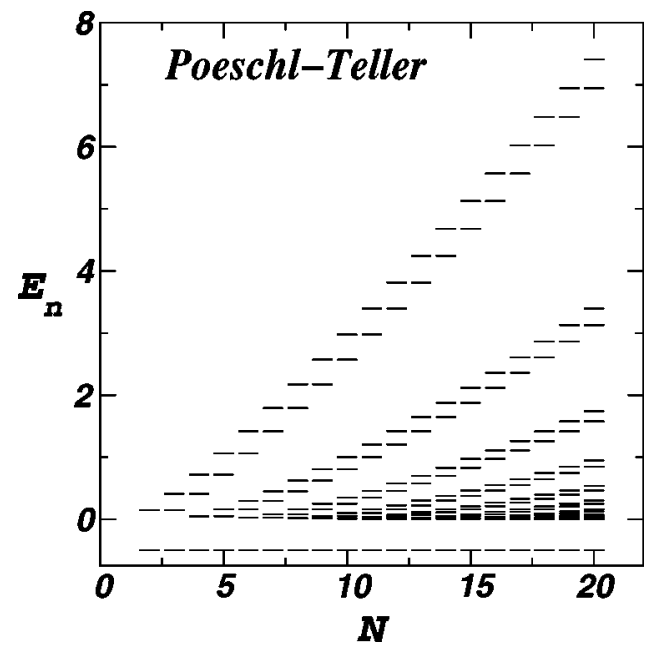

FIG. 3. Eigenvalues of the Poeschl-Teller Hamiltonian in the THO basis with dimensions ranging from $N=2$ to 20 .

mension of the THO basis increases, new energy levels appear. On the one hand, many of them lie close to the zero energy, increasing the level density in the region. On the other hand, new levels explore higher energies.

The wave functions obtained are orthonormal (see Fig. 4 where we present the $N=5$ case) and, as expected, with increasing energy they extend to higher $x$ ranges while the nodes accumulate in the vicinity of the origin. They have the desired asymptotic behavior and their nature allows for a straightforward use in calculations.

Once the eigenvalues and eigenfunctions of the Hamiltonian are obtained, we proceed to check convergence and closure of the truncated basis, calculating the total strength, energy weighted sum rule, and polarizability for a typical long-range operator $(x)$ and a short-range one [the potential $v(x)]$. The results obtained are presented in Tables I and II, respectively.

In Table I we include only even $N$ values. The odd $N$ +1 cases give identical result since the negative parity states are the only ones connected to the ground state due to the antisymmetric nature of the $x$ operator. The operator $v(x)$ is

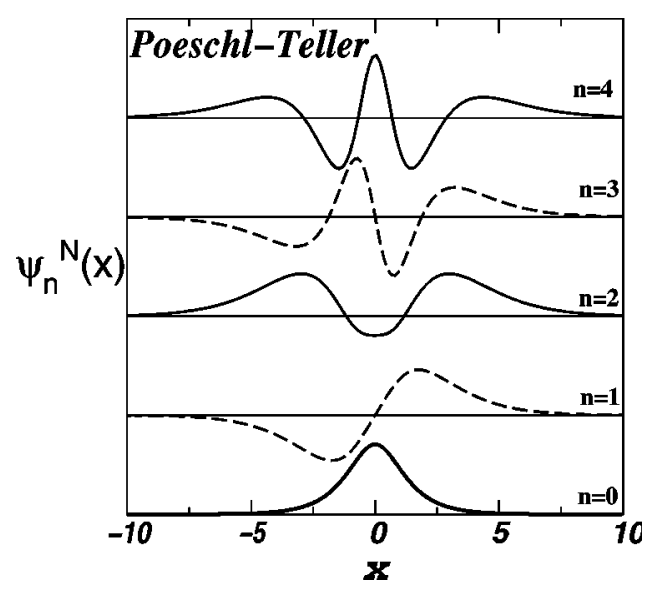

FIG. 4. Eigenstates ( $n=0$ to 4 ) of the Poeschl-Teller Hamiltonian diagonalized in the $N=5$ THO basis. 
TABLE I. Convergence of the total strength $\left(\mathcal{S}_{T}\right)$, energy weighted sum rule $\left(\mathcal{E}_{W}\right)$, and polarizability $(\mathcal{P})$ of the operator $x$ as a function of the THO basis dimension for the Poeschl-Teller Hamiltonian. $N$ is the total number of basis states. In this case, because of the parity selection rule, only odd parity states are connected to the ground state through the $x$ operator.

\begin{tabular}{rccc}
\hline \hline$N$ & $\mathcal{S}_{T}(x, N)$ & $\mathcal{E}_{W}(x, N)$ & $\mathcal{P}(x, N)$ \\
\hline 2 & 0.81577 & 0.52709 & 1.26254 \\
4 & 0.82245 & 0.50034 & 1.42050 \\
6 & 0.822467 & 0.49999 & 1.42344 \\
8 & & 0.50000 & 1.42349 \\
10 & & 0.50000 & 1.42350 \\
\hline Exact Value & 0.822467 & 0.50000 & 1.42350 \\
\hline \hline
\end{tabular}

symmetric and thus the situation is the opposite. Consequently, only odd $N$ values are shown in Table II.

In the long-range operator case the convergence is very fast for the three observables computed. For $N=4$ (three states in the continuum, only two with the right parity) we obtain the exact values within a $2 / 1000$ relative error. For the potential operator, the convergence is also fast although we need $N=17$ (eight states with the right parity) in the worst of the cases, to reach the same relative error as before. It is remarkable that the polarizability associated to the potential operator converges very rapidly. This indicates that perturbative corrections to the energy of the bound state, due to changes of the potentials, will be obtained accurately in this basis. In both cases we should stress the fast convergence obtained, which points out that the discretization performed is able to simulate correctly the continuum effects with the inclusion of few states in the THO basis.

\section{B. The Morse Hamiltonian}

The Morse potential [29] is a commonplace to model anharmonic vibrations in diatomic molecules [32] and it is be-

TABLE II. Convergence of the $\mathcal{S}_{T}, \mathcal{E}_{W}$ sum rule, and $\mathcal{P}$ of the operator $v(x)$ as a function of the THO basis dimension for the Poeschl-Teller Hamiltonian. $N$ is the total number of basis states. In this case, because of the parity selection rule, only even parity states are connected to the ground state through the $v(x)$ operator.

\begin{tabular}{rlll}
\hline \hline$N$ & $\mathcal{S}_{T}(v, N)$ & $\mathcal{E}_{W}(v, N)$ & $\mathcal{P}(v, N)$ \\
\hline 3 & 0.511992 & 0.061675 & 0.0739799 \\
5 & 0.527628 & 0.108383 & 0.0740682 \\
7 & 0.531714 & 0.132586 & 0.0740737 \\
9 & 0.532854 & 0.143771 & 0.0740741 \\
11 & 0.533187 & 0.148694 & \\
13 & 0.533287 & 0.150812 & \\
15 & 0.533318 & 0.151713 & \\
17 & 0.533328 & 0.152096 & \\
\hline Exact Value & 0.533333 & 0.152381 & 0.0740741 \\
\hline \hline
\end{tabular}

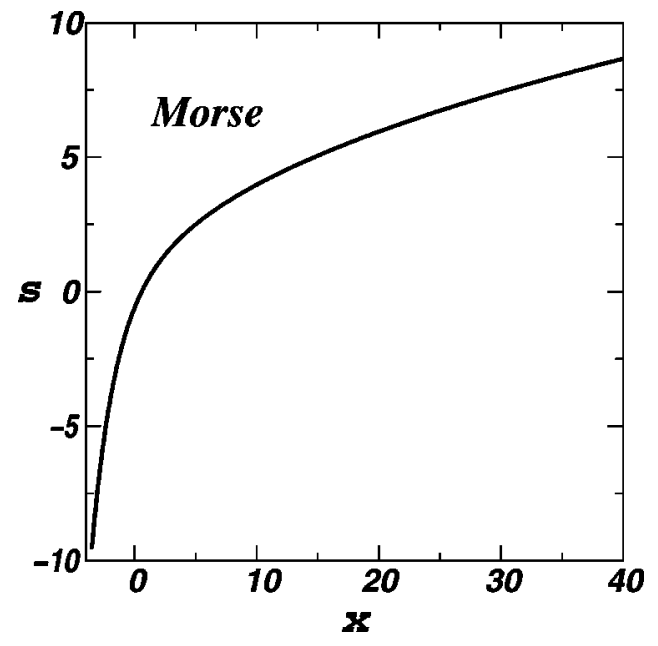

FIG. 5. Function $s(x)$ for the Morse Hamiltonian characterized by $j=1$.

coming of wide use in polyatomic structure calculations through the local mode picture $[33,34]$. The form of the potential is

$$
v(x)=D\left\{[1-\exp (-x)]^{2}-1\right\}
$$

where $x=\alpha r$, with $r$ the relative coordinate and $\alpha$ the inverse of the potential range, and $D$ is the potential depth in the minimum $(x=0)$. $D$ can be written in terms of a parameter $j[35]$, which is a positive real number, as

$$
D=\frac{1}{2}\left(j+\frac{1}{2}\right)^{2} .
$$

The bound wave functions for the Morse potential are written as

$$
\Psi_{j v}(x)=\mathcal{N}_{j v} \exp (-z / 2) z^{j-v} L_{v}^{2 j-2 v}(z)
$$

where $v$ is an integer number taking values from 0 up to the integer part of $j, \mathcal{N}_{j v}=\sqrt{(2 j-2 v) v ! /(2 j-v) !}$ is a normalization constant, $z=(2 j+1) \exp (-x)$ is the Morse variable, and $L_{s}^{(p)}(z)$ are the generalized Laguerre polynomials of degree $s$ and order $p$. As in the previous case we take $j=1$. The only true bound state in this case, $v=0$, has energy $e_{B}=$ $-\frac{1}{2}$ and its wave function is

$$
\psi_{B}^{M}(x)=3 \exp (-x) \exp [-3 \exp (-x) / 2]
$$

For $v=1$ there is another state that is not normalizable and corresponds to a resonance in the continuum at zero energy.

Direct integration in Eq. (7) provides the relation between $x$ and $s$ :

$$
[1+\operatorname{erf}(s)] / 2=[1+3 \exp (-x)] \exp [-3 \exp (-x)] .
$$

Numerically solving this equation we get the $s(x)$ function plotted in Fig. 5. The behavior of $s(x)$ reflects the potential 


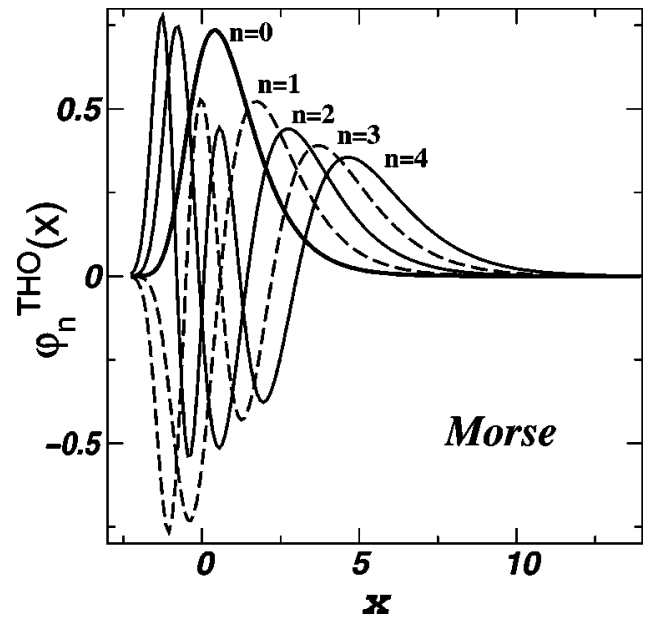

FIG. 6. Basis of THO for the Morse Hamiltonian, from $n=0$ to 4 .

asymmetry. Once the $s(x)$ function is computed we can define the THO basis following Eq. (5). The result for $N=5$ is depicted in Fig. 6.

The Hamiltonian diagonalization in the THO basis provides with eigenvalues and eigenfunctions. We plot in Fig. 7 the energies obtained increasing the dimension of the basis from $N=2$ to 20 . The bound-state energy lies at its exact value, $e_{B}=-\frac{1}{2}$, while the behavior of the positive eigenvalues is similar to the preceding case, excluding the appearance of parity doublets. Note the different scaling in Figs. 3 and 7, which shows the different behavior of the Poeschl-Teller and Morse potentials.

The eigenfunctions (see Fig. 8) form an orthonormal set. They are not symmetric, as expected, but as in the previous case, they both increase the number of nodes in the region around the origin and explore higher $|x|$ values as $n$ increases. Positive values of $x$ are explored much more rapidly as a function of $n$ than the negative ones.

With the obtained eigenvalues and eigenfunctions, we again check the convergence and closure of the truncated basis calculating the total strength, energy weighted sum

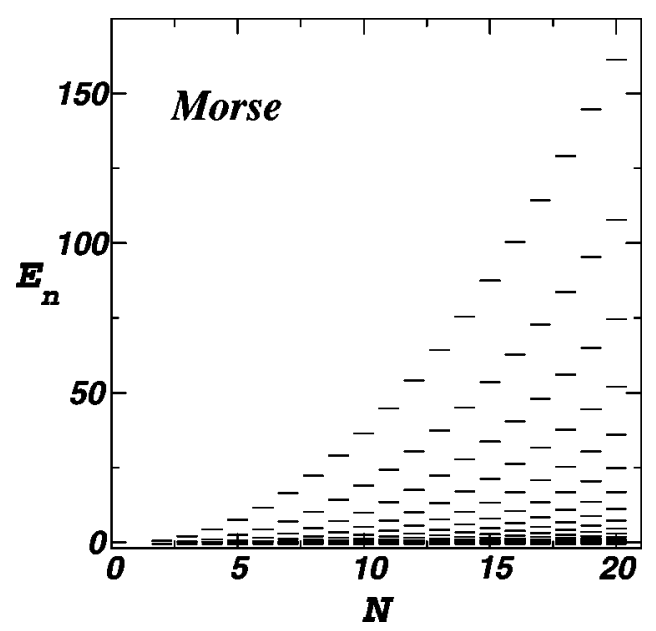

FIG. 7. Eigenvalues of the Morse Hamiltonian in the THO basis with dimensions ranging from $N=2$ to 20 .

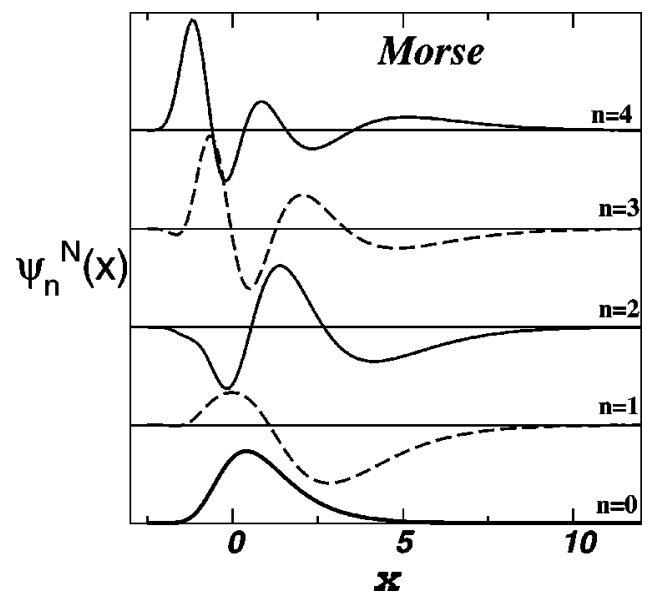

FIG. 8. Eigenstates ( $n=0$ to 4 ) of the Morse Hamiltonian diagonalized in the $N=5$ THO basis.

rule, and polarizability for the $x$ operator and the potential $v$. The results obtained are presented in Tables III and IV. In this case we cannot make any symmetry simplification.

In Table III the results for the $x$ operator are presented, showing a very fast convergence for all the computed observables. For $N=5$ (four states in the continuum) we obtain around 1/1000 maximum relative error. For the potential operator (see Table IV) with $N=6$ the maximum relative error is around 1/1000. Also, in this case, the convergence of the polarizability associated to the potential operator is very fast.

As in the Poeschl-Teller potential we should stress the fast convergence obtained, even faster in this case. That supports the evidence for considering the truncated THO basis as a suitable tool for continuum discretization.

\section{SUMMARY, CONCLUSIONS, AND OUTLOOK}

In this paper a THO basis has been introduced to produce appropriate normalizable states for discretizing the continuum. This is a fundamental problem in quantum mechanics and is especially relevant when treating weakly bound systems. The THO basis used in this paper is obtained by a local scale transformation (LST) that converts the ground state of the system into the harmonic-oscillator (HO) ground state. Thus the only previous requirement to apply this for-

TABLE III. Convergence of the $\mathcal{S}_{T}, \mathcal{E}_{W}$ sum rule, and $\mathcal{P}$ of the operator $x$ as a function of the THO basis dimension for the Morse Hamiltonian. $N$ is the total number of basis states.

\begin{tabular}{cccc}
\hline \hline$N$ & $\mathcal{S}_{T}(x, N)$ & $\mathcal{E}_{W}(x, N)$ & $\mathcal{P}(x, N)$ \\
\hline 2 & 1.08207 & 0.59344 & 0.658937 \\
3 & 1.10099 & 0.50068 & 0.894608 \\
4 & 1.10167 & 0.50004 & 0.950650 \\
5 & 1.10168 & 0.50000 & 0.960174 \\
6 & & & 0.961193 \\
7 & & & 0.961235 \\
8 & & & 0.961235 \\
\hline Exact Value & 1.10168 & 0.50000 & 0.961237 \\
\hline \hline
\end{tabular}


TABLE IV. Convergence of the $\mathcal{S}_{T}, \mathcal{E}_{W}$ sum rule, and $\mathcal{P}$ of the operator $v(x)$ as a function of the THO basis dimension for the Morse Hamiltonian. $N$ is the total number of basis states.

\begin{tabular}{llll}
\hline \hline$N$ & $\mathcal{S}_{T}(v, N)$ & $\mathcal{E}_{W}(v, N)$ & $\mathcal{P}(v, N)$ \\
\hline 2 & 0.575235 & 0.120856 & 0.0134195 \\
3 & 0.669899 & 0.178806 & 0.0936744 \\
4 & 0.740342 & 0.689425 & 0.0937489 \\
5 & 0.749840 & 0.919480 & 0.0937500 \\
6 & 0.749996 & 0.938555 & \\
7 & 0.750000 & 0.937510 & \\
8 & & 0.937500 & \\
\hline Exact Value & 0.750000 & 0.937500 & 0.0937500 \\
\hline \hline
\end{tabular}

malism is to know (either analytically or numerically) the ground state of the system. This defines the LST and allows to generate all the states in the THO basis by transforming the HO wave functions. The states in the THO basis are discrete, normalizable, and have exponentially decreasing asymptotic behavior. Although the basis is infinite, it is possible to get good approximations to the exact results when calculating observables of interest by truncating the basis to few states in the continuum region. In the calculations presented in this paper, truncating just to 7 or 8 states in the continuum gives the exact results within around one per mil relative error in the worst of the cases.

In this paper we have presented the formalism for onedimensional potentials and we have chosen the case of just one bound state. However we have performed calculations for several bound states and the same kind of results are obtained. The THO basis converge very rapidly to the exact energies of the bound eigenstates while states in the continuum lie close to zero energy, increasing the level density in that region, and few of them explore higher-energy regions.

The formalism presented here can be of use whenever bound states close to the dissociation limit are concerned or in the cases in which the coupling between bound and continuum states are important. It can be used for structure calculation to evaluate strength functions into the continuum and to perform scattering calculations taking into account the breakup effects.
The use of THO wave functions in practical calculations involves increasing the number $N$ of states considered in the calculation until convergence is achieved. In this sense, the THO basis has an advantage over the use of a box to calculate continuum effects, because, in this case, one has to deal with two continuum parameters, which are the radius of the box, and the maximum energy of the states considered. Thus, it is much harder to demonstrate convergence when there are two parameters to vary, instead of just one discrete parameter.

The CDCC calculations have similar convergence problems. The bins describing continuum discretization are such that when expressed in terms of the coordinates, they vanish at distances of the order of $1 / \Delta k$. A practical CDCC calculation requires to fix the maximum $k$ value considered and the interval $\Delta k$ of the bins, so that the number of bins is given by $N=k_{\max } / \Delta k$. Here, also to demonstrate convergence, one has to deal with two parameters. In addition, the CDCC method requires to solve the Schrödinger equation for all the energies in the continuum. In our case, there is only one discrete parameter to check convergence and it is only required to solve the Schrödinger equation for the ground state.

The THO basis also has some similarities with the Sturmian basis. In both cases the basis is discrete and normalizable, and the wave functions have the same asymptotic behavior as the ground state. However, the Sturmian basis requires solving the Schrödinger equation for increasing values of the potential depth, obtaining in this way wave functions with the same energy, but more nodes. These wave functions are not orthogonal, as they correspond to different Hamiltonians. Besides, the Sturmian basis gives an accurate description of the interior of the potential, but it converges very slowly to describe large separations. Thus, the THO basis has the advantage that one has to solve only the Schrödinger equation for the ground state, the wave functions are orthogonal, and the description of distances beyond the potential range seems to be satisfactory.

\section{ACKNOWLEDGMENTS}

This work was supported in part by the Spanish DGICYT under Project Nos. PB98-1111 and FPA2000-1592-C03-02. We acknowledge useful discussions with F. Iachello, P. H. Vaccaro, A. Frank, R. Lemus, and R. C. Johnson.
[1] M.V. Stoitsov, P. Ring, D. Vretenar, and G.A. Lalazissis, Phys. Rev. C 58, 2086 (1998).

[2] M.V. Stoitsov, W. Nazarewicz, and S. Pittel, Phys. Rev. C 58, 2092 (1998).

[3] M.V. Stoitsov, J. Dobaczewski, P. Ring, and S. Pittel, Phys. Rev. C 61, 034311 (2000).

[4] N. Sandulescu, Nguyen Van Giai, and R.J. Liotta, Phys. Rev. C 61, 061301 (2000).

[5] S. Mizutori, J. Dobaczewski, G.A. Lalazissis, W. Nazarewicz, and P.-G. Reinhard, Phys. Rev. C 61, 044326 (2000).

[6] K. Bennaceur, J. Dobaczewski, and M. Ploszajczak, Phys.
Rev. C 60, 034308 (1999).

[7] I. Cacelli, R. Moccia, and A. Rizzo, Phys. Rev. A 57, 1895 (1998).

[8] T.N. Rescigno, A.E. Orel, and C.W. McCurdy, Phys. Rev. A 55, 342 (1997).

[9] A.K. Kazansky, J. Phys. B 29, 4709 (1996).

[10] J.M. Vogels, B.J. Verhaar, and R.H. Blok, Phys. Rev. A 57, 4049 (1998).

[11] M.B. Campbell, T.J. Bensky, and R.R. Jones, Phys. Rev. A 57, 4616 (1998).

[12] B.I. Schneider, Phys. Rev. A 55, 3417 (1997). 
[13] A.K. Kazansky, J. Phys. B 30, 1401 (1997).

[14] A.C. Mueller et al., Nuclear Physics in Europe: Highlights and Opportunities, edited by J. Vervier et al., NuPECC Report, 1997, p. 31.

[15] E.P. Wigner and L. Eisenbud, Phys. Rev. 72, 29 (1947); Atomic and Molecular Processes: An R-Matrix Approach, edited by P.G. Burke and K.A. Berrington (IOP, Bristol, 1993).

[16] M. Rotenberg, Adv. At. Mol. Phys. 6, 233 (1970).

[17] F. Antonsen, Phys. Rev. A 60, 812 (1999).

[18] R. Szmytkowski and B. Zywicka-Mozejko, Phys. Rev. A 62, 022104 (2000).

[19] O.I. Tolstikhin, V.N. Ostrovsky, and H. Nakamura, Phys. Rev. A 58, 2077 (1998).

[20] R.G. Lovas, R.J. Liotta, A. Insolia, K. Varga, and D.S. Delion. Phys. Rep. 294, 265 (1998).

[21] N. Austern, Y. Iseri, M. Kamimura, M. Kawai, G. Rawitsher, and M. Yahiro, Phys. Rep. 154, 125 (1987).

[22] M. Moshinsky and Y. Smirnov, The Harmonic Oscillator in Modern Physics (Harwood Academic, Amsterdam, 1996).

[23] I.Zh. Petkov and M.V. Stoitsov, C. R. Acad. Bulg. Sci. 34, 1651 (1981).
[24] I.Zh. Petkov and M.V. Stoitsov, Theor. Math. Phys. 55, 584 (1983).

[25] I.Zh. Petkov and M.V. Stoitsov, Yad. Fiz. 37, 1167 (1983) [Sov. J. Nucl. Phys. 37, 692 (1983)].

[26] M.V. Stoitsov and I.Zh. Petkov, Ann. Phys. (N.Y.) 184, 121 (1988).

[27] I.Zh. Petkov and M.V. Stoitsov, Nuclear Density Functional Theory, Oxford Studies in Physics (Clarendon, Oxford, 1991).

[28] G. Pöschl and E. Teller, Z. Phys. 83, 143 (1933).

[29] P.M. Morse, Phys. Rev. 34, 57 (1929).

[30] O. Bohigas, A.M. Lane, and J. Martorell, Phys. Rep. 51, 267 (1979).

[31] Y. Alhassid, F. Gursey, and F. Iachello, Ann. Phys. (N.Y.) 148, 346 (1983).

[32] G. Herzberg, Molecular Spectra and Molecular Structure, Vol 1: Spectra od Diatomic Molecule (Van Nostrand Reinhold, NY, 1966).

[33] M.S. Child and L. Halonen, Adv. Chem. Phys. 57, 1 (1984).

[34] J.K. Watson, Chem. Phys. 190, 291 (1995).

[35] F. Iachello and R.D. Levine, Algebraic Theory of Molecules (Oxford University, Oxford, 1995). 